\title{
Endovascular Management of Cerebral Aneurysms: Work in Progress
}

Can. J. Neurol. Sci. 2007; 34: 1-2

It has been several years since the first publication of the international subarachnoid aneurysm trial (ISAT) results, ${ }^{1}$ and medium-term follow-up (with survival curves to seven years) have been reported more recently. ${ }^{2}$ In ISAT, $>70 \%$ of eligible patients were selectively excluded by criteria that were never disclosed. The remaining 2143 primarily good-grade patients with ruptured aneurysms (mostly small and located in the anterior circulation) were randomized between aneurysm clipping and endovascular coiling. At one year there was no difference in fatality rates between the two treatment groups, but for combined death and dependent rates there was an absolute risk reduction of $6.9 \%$ associated with coiling, which was significant. Endovascular treatment has been accepted by most to be the preferred treatment modality for basilar artery aneurysms, which are less common and difficult to repair surgically, so the main impact of ISAT has been to contribute to a steady increase in the use of endovascular coils in the management of anterior circulation aneurysms, depending on the availability of endovascular expertise at individual centers. For reasons alluded to above ISAT results are not conclusive, but accumulating clinical experience confirms that early and midterm results following endovascular repair can be good. The major worry about endovascular coiling remains incomplete aneurysm obliteration (more common in large, complex and wide-necked aneurysms) and the risk associated with remnant growth or aneurysm recanalization, seen in up to one-third of treated aneurysms over several years, and subsequent rebleeding. ${ }^{3-5}$ This risk mandates long-term follow-up, repeat angiography and in many patients retreatment of their aneurysm with either more coiling or microsurgery along with the attendant risks of these interventions. With those concerns in mind, the CARAT investigators (Cerebral Aneurysm Rerupture After Treatment) have recently reported the results of a prospective cohort study of 1010 subarachnoid hemorrhage patients, of whom 711 underwent microsurgical clipping and 299 coiling, with 904 patient-years follow-up. ${ }^{6}$ Rerupture was rare in both treatment groups, but as expected retreatment was required for a percentage of patients who had undergone coiling, performed in almost $8 \%$ of the patients in this group the first year following treatment, $4.5 \%$ in the second and $3.5 \%$ in the third year, compared to $1.7 \%$ of surgically clipped patients requiring retreatment in follow-up $(p<0.0001)$. On a very positive note, however, it was found that retreatment was rarely associated with major complications in CARAT.

Despite CARAT's encouraging results it would still be preferable to reduce the need for follow-up and possible retreatment of coiled aneurysms, anxious events for patients and associated with at least some risk. One of the first coils designed and introduced to help tackle the problem of aneurysm recurrence was the HydroCoil, a platinum wire coated with a layer of hydrophilic polymer which swells when in contact with blood resulting in a greater density of packing in the aneurysm sac. Although it is intuitive that increased packing should reduce the risk of aneurysm reopening, this theory has not been addressed in clinical studies until recently.

The work reported by Gunnarsson et $\mathrm{al}^{7}$ in this edition of the Canadian Journal of Neurological Sciences provides some clinical support to the premise of HydroCoil aneurysm treatment. The experienced group of neurointerventionalists at the Toronto Western Hospital selected 28 patients with 29 aneurysms for Hydrocoil treatment over a roughly one year period during which time we are told the eligibility for Hydrocoil treatment relaxed as they overcame issues such as the relative "stiffness" of Hydrocoils and the need for larger microcatheters through which the coils are delivered. (A number of important technical tips and suggestions regarding the use of Hydrocoils are included in this report.) Seventeen (61\%) of the patients had ruptured aneurysms, 25 had saccular-type aneurysms (three others were dissecting and another was a pseudoaneurysm), none were less than $5.5 \mathrm{~mm}$ in maximal diameter, and as might be expected several coil combinations and techniques were employed as required by individual aneurysm characteristics. Of the 25 saccular aneurysms where Hydrocoils were used and at a mean follow-up of roughly three months, $44 \%$ were completely occluded, $36 \%$ had a residual neck and $20 \%$ had residual aneurysm filling. The calculated mean aneurysm packing in this group was $76 \%$, and while we do not have an institutional cohort of patients to compare with in this study, the authors point out this figure compares quite favourably with bare platinum wire packing volumes described in the literature. Whether the surrogate outcome of "packing density" correlates with long-term aneurysm stability and aneurysm recurrence rates has yet to be established; the length of follow-up of this study is too short to draw any conclusions. And while the use of non-invasive ATECO MRA to search for aneurysm recurrences in this study is appealing, it could be argued that accurate determination of recurrence rates following Hydrocoil treatment should be done with digital subtraction angiography-still the gold standard. Only two serious, consequential complications occurred in the Hydrocoil treated patients.

Encouraging Hydrocoil results have also been recently reported by Gaba et $\mathrm{al}^{8}$ who matched 50 Hydrocoil-treated aneurysms to 57 similar aneurysms treated with bare platinum coils. The Hydrocoil cohort was associated with a significantly greater volumetric packing occlusion ( $85 \%$ vs. $30 \%)$, a reduced one year aneurysm recurrence rate (17\% vs. $24 \%)$, and no 
incidences of postembolization rupture from coil mass expansion, worsened mass effect, or asceptic meningitis (all concerns that have been voiced regarding the use of Hydrocoils).

Coil technology will improve beyond the bare platinum wire. Efforts to understand the molecular mechanisms of aneurysm healing are underway. Encouraging results in animals suggest that "radioactive" coils ( ${ }^{32} \mathrm{P}$ impregnated coils) might reduce the risk of recanalization without short-term risks, ${ }^{9}$ but a bioactive coil also designed to enhance intra-aneurysmal clot organization and fibrosis (Matrix detachable coils; Boston Scientific Neurovascular) has not yet been shown to be superior to bare platinum coils when used in humans. ${ }^{10,11}$ Bioactive endovascular technology has also examined the delivery of growth factors, ${ }^{12,13}$ gene therapy ${ }^{14}$ and cellular substrates ${ }^{15}$ on cellular growth associated with coil embolization in experimental models.

Endovascular coiling is an elegant technical procedure that is steadily supplanting microsurgical clipping for the ablation of cerebral aneurysms. There is no contest between the two treatments when "invasiveness" and short-term procedure-related morbidity are compared, providing coiling is performed by skilled interventionalists in suitable patients. However there remain a number of clinical circumstances where other considerations must be taken into account, such as large intracerebral hematomas with associated mass effect requiring surgical evacuation, and large volume subarachnoid or intraventricular hemorrhages that might be treated with fibrinolysis. Should patients such as these be coiled leaving unattended morbid intracranial hematomas, or should their hematomas and aneurysms be dealt with in a staged fashion? The practical approach up until now has been surgery for aneurysm clipping combined with clot treatment. What about patients in whom a good or even reasonable coil occlusion is uncertain or poor based on aneurysm neck width or dome morphologyshould coiling, as the less invasive alternative to surgery, be carried out as matter of routine, with a higher likelihood of the patient being left with less than satisfactory treatment and a now chronic, rather than cured condition? Finally, for patients in very poor neurological condition following aneurysm rupture, clearly not candidates for open surgery but for whom we might feel compelled to "take a chance", our temptation is to quickly protect the aneurysm dome with coils and then pray for a miracle. Is that good for patients and their families and is it an appropriate use of our health care resources?

In spite of being marketed as the modern and better solution to cerebral aneurysms endovascular coiling requires (and is undergoing) further study, particularly in terms of long-term efficacy and durability. Before we ride the coil pendulum too far we need more evidence establishing the long-term durability of endovascular aneurysm repair, particularly in those patients in whom a remnant is appreciated. The endovascular management of cerebral aneurysms is indeed a work in progress, and in the meantime microsurgical clip repair with intraoperative or postoperative angiography to confirm complete aneurysm ablation remains the right choice for many patients in whom a good recovery and long life following are possible.

\section{REFERENCES}

1. Molyneux A, Kerr R, Stratton I, Sandercock P, Clarke M, Shrimpton J, et al. International Subarachnoid Aneurysm Trial (ISAT) of neurosurgical clipping versus endovascular coiling in 2143 patients with ruptured intracranial aneurysms: a randomised trial. Lancet. 2002;360(9342):1267-74.

2. Molyneux AJ, Kerr RS, Yu L-M, et al. International Subarachnoid Aneurysm Trial (ISAT) of neurosurgical clipping versus endovascular coiling in 2143 patients with ruptured intracranial aneurysms: a randomised comparison of effects on survival, dependency, seizures, rebleeding, subgroups and aneurysm occlusion. Lancet. 2005;366:809-17.

3. Murayama Y, Nien YL, Duckwiler G, Gobin YP, Jahan R, Frazee J, Martin N, Vinuela F. Guglielmi detachable coil embolization of cerebral aneurysms: 11 years' experience. J Neurosurgery. 2003;98:959-66.

4. Raymond J, Guilbert F, Weill A, Georganos SA, Juravsky L, Lambert A, et al. Long-term angiographic recurrences after selective endovascular treatment of aneurysms with detachable coils. Stroke. 2003;34:1398-403.

5. Van der Schaaf I, Algra A, Wermer MJ, Molyneux A, Clarke M, van Gijn J, et al. Endovascular coiling versus neurosurgical clipping for patients with aneurysmal subarachnid hemorrhage. Stroke. 2006;37:572-3

6. The CARAT Investigators. Rates of delayed rebleeding from intracranial aneurysms are low after surgical and endovascular treatment. Stroke. 2006;37:1437-42.

7. Gunnarsson T, Klurfan P, terBrugge KG, Willinsky RA. Treatment of intracranial aneurysms with hydrogel coated expandable coils. Can J Neurol Sci. 2007;34:38-46.

8. Gaba RC, Ansari SA, Roy SS, Marden FA, Viana MAG, Malisch TW. Embolization of intracranial aneurysms with hydrogelcoated coils versus inert platinum coils. Effects on packing density, coil length and quantity, procedure performance, cost , length of stay and durability of therapy. Stroke. 2006;37: 1443-50.

9. Raymond J, Mounayer C, Salazkin I, Metcalfe A, Gevry G, JanickiC, et al. Safey and effectiveness of radioactive coil embolization of aneurysms. Effects of radiation on recanalization, clot organization, neointima formation, and surrounding nerves in experimental models. Stroke. 2006; 37:2147-52.

10. Taschner CA, Leclerc X, Rachdi H, Barros AM, Pruvo J-P. Matrix detachable coils for the endovascular treatment of intracranial aneurysms. Analysis of early angiographic and clinical outcomes. Stroke. 2005;36:2176-80.

11. Niimi Y, Song J, Madrid M, Berenstein A. Endosaccular treatment of intracranial aneurysms using matrix coils. Early experience and midterm follow-up. Stroke. 2006;37:1028-32.

12. Abrahams JM, Forman MS, Grady MS, Diamond SL. Delivery of human vascular endothelial growth factor with platinum coils enhances wall thickening and coil impregnation in a rat aneurysm model. AJNR. 2001;22:1410-17.

13. Ohyama T, Nishide T, Iwata H, Sato H, Toda M, Toma N, et al. Immodibization of basic fibroblast growth factor on a platinum microcoil to enhance tissue organization in intracranial aneurysms. J Neurosurg. 2005;102:109-15.

14. Abrahams JM, Song C, DeFelice S, Grady MS, Diamond SL, Levy RJ. Endovascular microcoil gene delivery using immobilized anti-adenovirus antibody for vector tethering. Stroke. 2002; 33:1376-82.

15. Kawakami O, Miyamoto S, Hatano T, Yamada K, Hashimoto N, Tabata Y. Accelerated embolization healing of aneurysm by polyethelye terephthalate coils seeded with autologous fibroblasts. Neurosurgery. 2005;56:1075-81. 